\title{
The Implementation and Application of Data Visualization Based on CAS-MES
}

\author{
Jinhua Zhu', a , Peng $\mathrm{Li}^{2, \mathrm{~b}}$ \\ ${ }^{1}$ Shenyang Institute of Automation, Chinese Academy of Sciences \\ Research Laboratory of Digital Factory, Shenyang, 110179, China \\ ${ }^{2}$ Shenyang Institute of Automation, Chinese Academy of Sciences \\ Research Laboratory of Digital Factory, Shenyang, 110179, China \\ aemail: zhujinhua@sia.cn, bemail: pengli@sia.cn
}

Keywords: CAS-MES; Data Visualization; Visual Modeling; Visual Monitoring

\begin{abstract}
Data visualization plays more and more important roles in the process of information technology popularization among the enterprises. With the enlargement of the valid data acquired in daily production, great challenges of data display for the users and utilization of the data, which requires scientific analysis to discover inherent connection, are formed. As a result, in order to improving the data utilization and promoting the amity between users and platform, the implementation and application of data visualization based on CAS-MES is discussed, which is aimed at leading a new way to effective management for the enterprises.
\end{abstract}

\section{Introduction}

As the expansion of the business and the improvement of the information technology [1][2] implemented in the enterprises go along, massive data has been produced continually. Generally, these data is stored in the database and will be used and displayed in the form of sheets when it is needed to. Sheets, one of the diverse display form, play an important role in the data display while there follow the disadvantages, for instance:

(1) Data display [3] in the form of sheets may work in the simple business, but it is not so convenient for users to dig information through data sheets when the relative business is complicated. Thus, hardly can friendly interface be achieved under this circumstance.

(2) On the other hand, direct and comprehensive data display in the form of sheets is at the price of software complicity and proportionate increase in development work.

(3) Moreover, not only is the data acquired in daily production used in display, but it is also used in analysis, comparison and connection in order to predict the parameter trend. As a result, the data in the form of sheets is lack of corresponding functions.

According to the reasons above, single data display in the form of sheets hardly meets the need of industrial production. With the development of science and technology and successful implementation in CAS-MES, data display in the form of visualization [4][5] shows great advantages as follows, which make it important and necessary:

(1) Data display in the form of visualization [6] enriches the expressive mode of the data according to users needs. Not only does the combination usage of sheets, pie diagram and bar diagram [7] make the interface friendly, but also simplifies the development for programmers.

(2) Interactions between users and platform will become easier and more convenient under the circumstance of data visualization. The operations such as data searching, saving or editing will be shorten from multiple steps to even one.

(3) Multiple-dimension [8] data can be classified, ordered, combined and displayed according to the properties of the objects, which will be used in analysis, comparison and connection.

CAS-MES, which has been put into use [9] for decades, has already proved efficiency. Many functions of CAS-MES, especially visual Modeling, base on the idea of data visualization and show great advantages in modeling, display and analysis, making CAS-MES a successful and competitive 
software.

\section{The Visual Modeling Process}

As the core business of CAS-MES and typical application of data visualization technology, visual modeling is applied to plant modeling, product modeling and procedure modeling. Considering the similarity in the structure and the achievement process of each modeling, visual modeling will be fully discussed below as a whole. The general process of visual modeling can be concluded as the flow chart as follows:

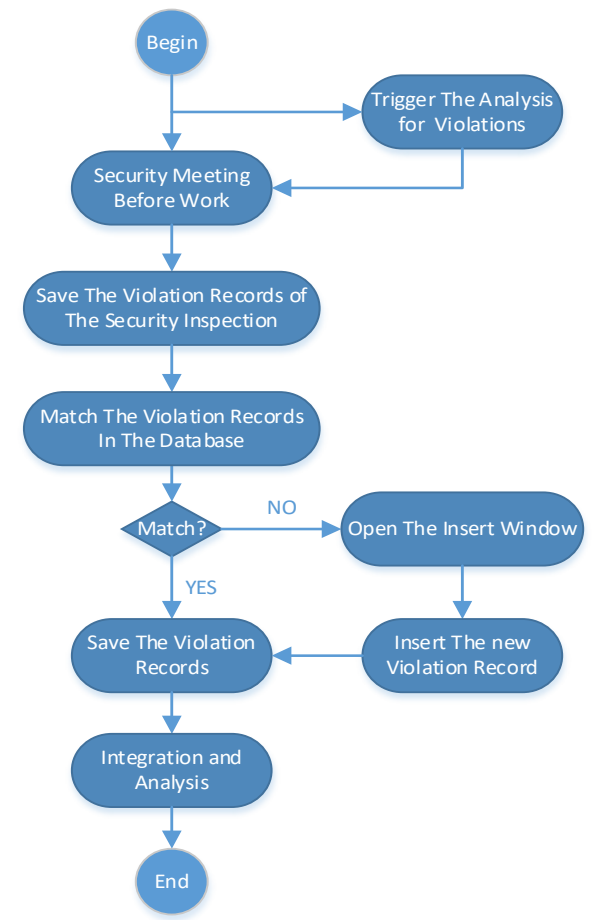

Fig.1. The General Process of Visual Modeling

As the flow chart shows, the process of visual modeling can be further subdivided into basic function designs and modeling tool designs, either of which the article will elaborate on below.

\section{Basic Functions Design}

(1) Basic Movement design for Basic Elements

The basic elements for modeling require basic functions such as moving, resizing and rotating. As the MSDN describes, the thumb control " represents a control that lets the user drag and resize controls", so it is a perfect candidate for the demand.

The process of the moving, resizing and rotating achievement can be summarized as follows:

- To create the class inherited from Thumb control with a proper class name;

- To Make sure proper implementation of the event handle;

- To develop the logic actions of relative functions within the proper event handler;

- To use the class with relative functions as the control required

(2) Appearance Design For Elements

Control Template and Data Template are the basic templates in WPF, which is short for Windows Presentation Foundation, as tools for appearance design of elements or controls. The former is the control template and the latter is the data template. The function of each template can be concluded as follows:

- Control Template

The visual appearance of a control, as such the shape of the button control or the background of the TextBox control, is mainly up to the design of the Control Template.

- Data Template

Data Template is a similar concept to Control Templates. A very flexible and powerful solution 
based on this template can be put forward to replace the visual appearance of a data item in a control.

(3) Connection Function Design For Elements

There exist different techniques to achieve connection function among the elements. The approach this article provided is that the elements themselves provide connection points from which the user can drag a connection to other elements.

First, move the mouse over a element and four visual elements called connectors;

Then click the left mouse button and start dragging, the connector will create a decoration which is an adorner of type ConnectorAdorner;

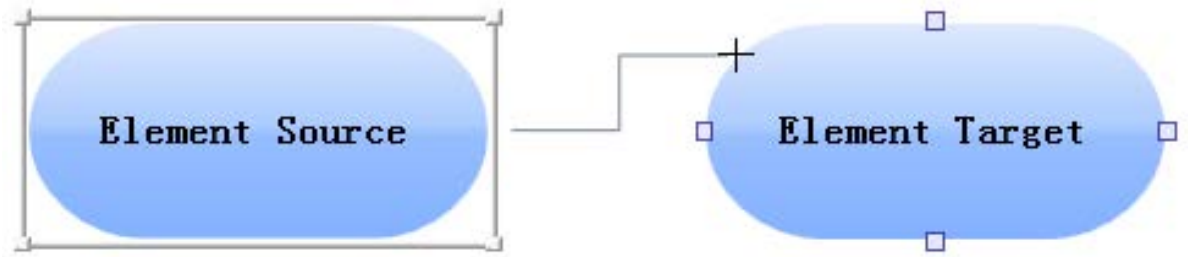

Fig.2. Searching for Sink Connector

Then release the mouse button over a connector, the ConnectorAdorner creates a new connection instance and adds it to the element. If the mouse button is released elsewhere, no connection instance is created.

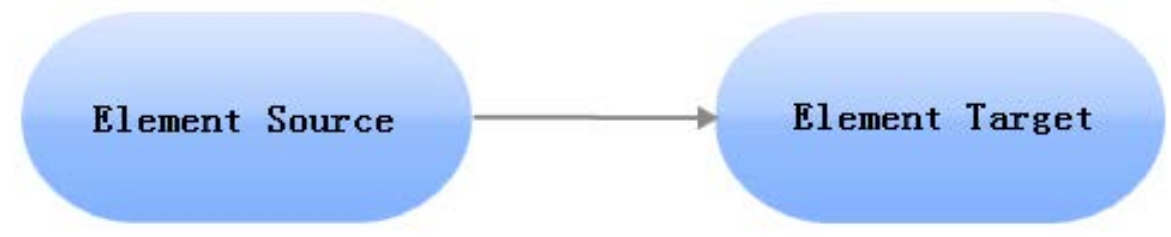

Fig.3. Finishing Connection

The Connection implements the ISelectable interface. If a connection instance is selected, two rectangles at each end of the connection path will show up;

Each of the two rectangles represents a Thumb control and they are part of a ConnectionAdorner instance which allows the user to modify existing connections. If the target element of the connection is dragged to another connector and release it there, the user can re-connect the existing connection.

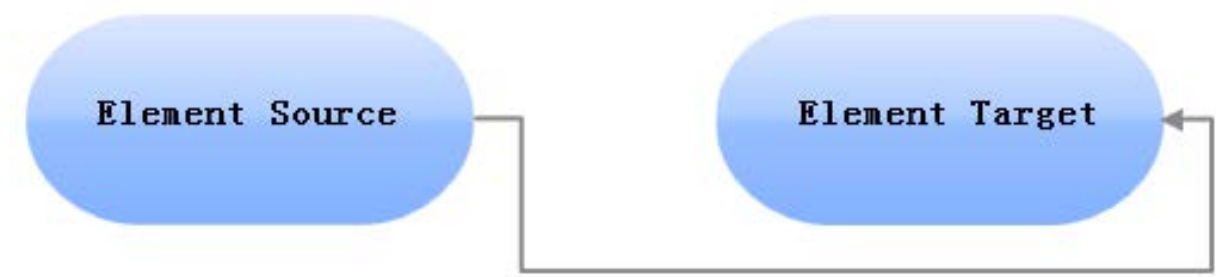

Fig.4. The result of Connector Selection

\section{Visual Modeling Tool Design}

In the previous chapter, the elements have been shown how to move, resize, rotate and interact with each other. With the functions, further features which is essential for the Modeling tool will be presented below.

(1) Design Canvas

Design canvas is where elements act and interact. Sometimes when the element is moved outside the border of the design canvas, it is found no longer accessible. Normally scroll bars are expected that any elements can easily scroll to outside the visible canvas region. But with the experiment of placing a control on a canvas and positioning it outside the boundaries of the Canvas, it finds that there is no change of the size of the canvas.

Why will the canvas keep its size, no matter where the control is placed?Of course not. The canvas will never notify the scroll event of the scroll bars just because there is none event of the 
scroll bars but of the control itself.

The solution is that the canvas must adjust its size every time when the element is moved or resized. Fortunately the canvas class provides an overrideable method named "MeassureOverride" that allows the canvas to calculate its desired size and return it to the WPF layout system, which means the canvas for modeling will be infinite in width and length.

(2) Tool Box

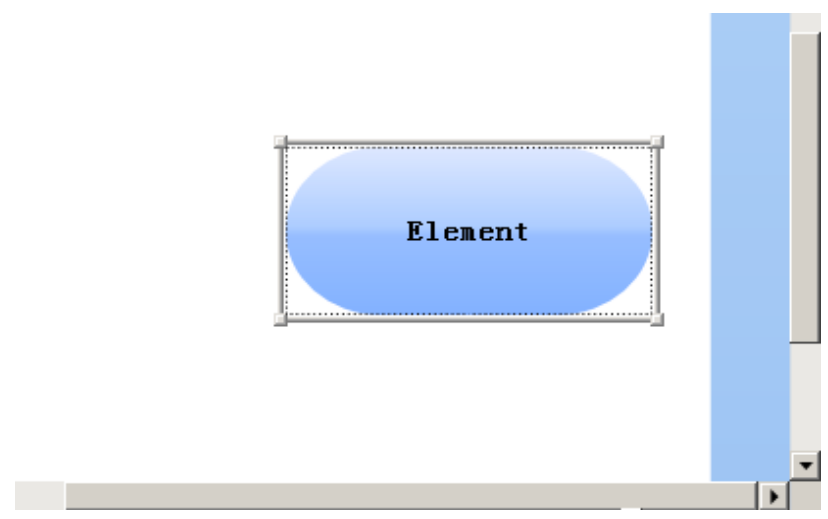

Fig.5. The Canvas Adapt to Elements Move

The canvas is work zone while the tool box is the inventory for elements. The tool box in programming is an ItemsControl that uses the ToolboxItem class which is defined as default container to display its elements. If an element is dragged from the tool box and dropped on the canvas, the relative events of the ToolboxItem will be triggered, which makes the ToolboxItem the start of the drag operations in programming. There is nothing mysterious about dragging and dropping elements, but attention should be still payed to how to copy an element from the drag source (Toolbox) to the drop target (canvas). One solution is to use the XamlWriter to serialize the content of the ToolboxItem into XAML. The achievement of tool box for elements is as the Fig. 8 shows.
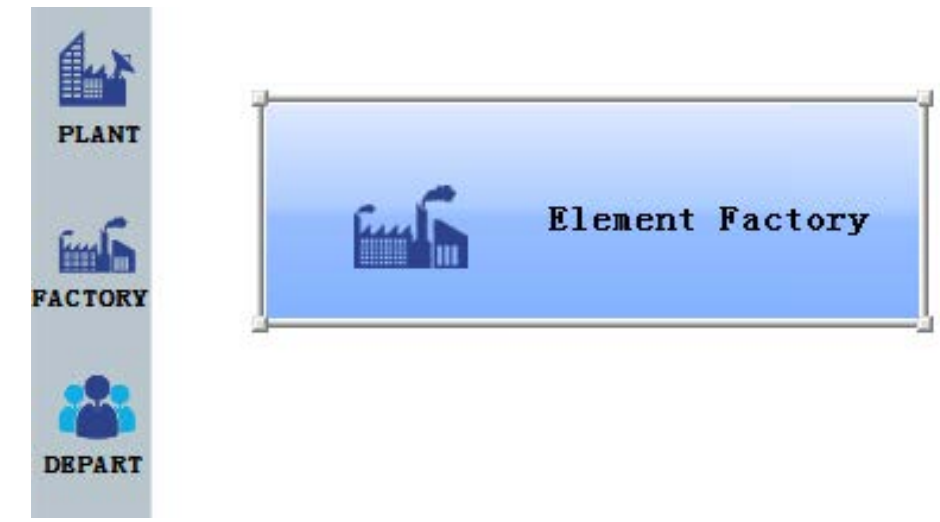

Fig.6. Achievement of Element Inventory Named Tool Box

\section{Application of Visual Modeling}

Considering there is a project which needs real time visual monitoring for the cargo transport during the production. The demands is summarized as follows:

- Real time visual monitoring for the process of cargo transport;

- Different tag for different transport status;

- Relative parameters statistics;

- Other interfaces for further purposes.

The solution presents from the first beginning of elements design is:

(1) The design of elements which delegate the devices

The first step of the visual monitoring for the cargo transport is the basic production elements design for the devices involved in the transport. The demand for this step is that the elements should 
be designed as resemble of its appearance as the real devices and convenient for appearance change such as the color, shape etc. Parts of elements designed for the devices are shown below:

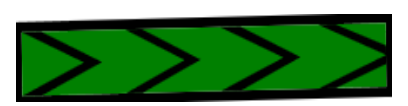

(a) Belt Conveyor

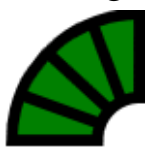

(b) Corner Belt

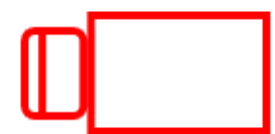

(c) Vehicles

Fig.7. Parts of the Elements Designed for the Devices

(2) The area design for the statistics

According to the project demand, the status of the transport parameters will be supervised at the same time with the visual modeling for the transport. So the parameters statistic design is as follows:

\begin{tabular}{|l|c|}
\hline Parameters Supervision \\
\hline Cargo Status \\
\hline Dea1 ing Number & 31400 \\
\hline Saturation & $30 \%$ \\
\hline Be1t Status \\
\hline Be1t Run No. \\
\hline Be1t Stop No. & 7 \\
\hline B1et B1ock No. & 0 \\
\hline
\end{tabular}

Fig.8. Parameters Statistic Design

(3) The modeling of visual monitoring for cargo transport

After the preparation of the elements design for the devices and the parameters statistic area design, the whole modeling of visual monitoring for the cargo transport will be achieved. The modeling is presented like this:

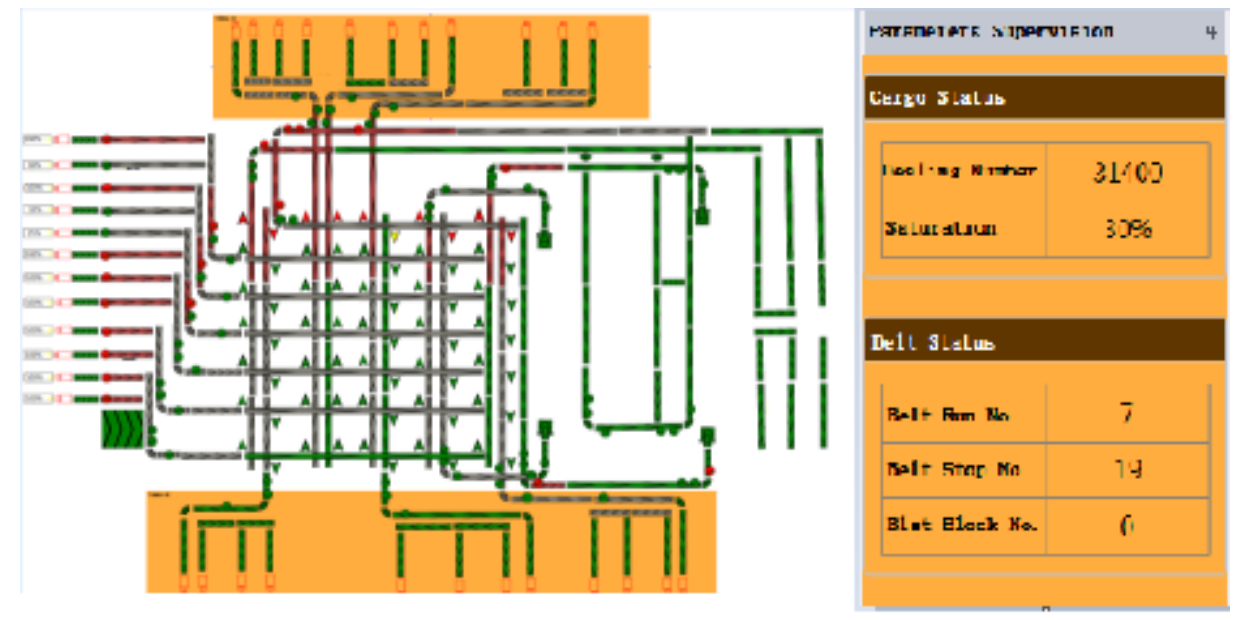

Fig.9. the Modeling of the Cargo Transport

(4) The mechanism of the visual monitoring

With the help of the data acquisition from the real time/historical database, the variation of the data will be presented in the modeling of the visual monitoring for the cargo transport. The mechanism of the visual monitoring meets the demand of the project:

- Real-time visual monitoring based on real-time data acquisition has been achieved;

- Different colors of the element represent different working status of the belt;

- Relative parameters statistic are easy to check through the statistics table;

- The interfaces for further functions are made. 


\section{Conclusion}

Data visualization plays more and more important roles in the process of information technology popularization among the enterprises. This article presents an method of visual modeling and the visual monitoring based on the modeling through implementation and application of data visualization technology from the basic functions and appearance design of the basic elements to the modeling accomplish until the visual monitoring achievement for the application of the project. Although the method presented by this article has been put into use in many projects for decades and has already proved its simplicity, convenience and efficiency, there is much improvement to do for the functions and the appearance design of basic elements or the operative convenience for the visual modeling though consummating the commands system and adding the shortcut operations especially customizing the functions for visual modeling. Optimistic outlook has already displayed with the development of the technology in data visualization and successful implementation with meeting the variation needs of different projects. It is the responsibility and great honor to make due contributions through much work for the innovation and improvement of the visual modeling and its application based on data visualization technology for the modernization of our country.

\section{Acknowledgement}

This work is supported by the Science and Technology Plan Project of Liaoning Province (2015106 015) and the Key Laboratory of Network Control System, Chinese Academy of Sciences.

\section{References}

[1] Kehrer, Johannes, Hauser, Helwig. Visualization and Visual Analysis of Multifaceted Scientific Data: A Survey [J]. IEEE transactions on visualization and computer graphics, 2013, 19 (3): 495-513.

[2] Fuchs, R, Hauser, H. Visualization of Multi-Variate Scientific Data [J]. Computer Graphics Forum: Journal of the European Association for Computer Graphics, 2009, 28 (6): 1670-1690.

[3] Min Chen, Ebert D., Hagen H. et al. Data, Information, and Knowledge in Visualization [J]. IEEE Computer Graphics and Applications, 2009, 29 (1): 12-19.

[4] Chao-Ying Chiu, Alan D. Russell. Design of a construction management data visualization environment: A top-down approach [J]. Automation in construction, 2011, 20 (4): 399-417.

[5] Tao Cheng, Jochen Teizer. Real-time resource location data collection and visualization technology for construction safety and activity monitoring applications [J]. Automation in construction, 2013, 34 (Sep.): 3-15.

[6] Heeyoul Choi. Data visualization for asymmetric relations [J]. Neurocomputing, 2014, 124 (Jan. 26): 97-104.

[7] Borji, A., Sihite, D. N. , Itti, L. et al. Quantitative Analysis of Human-Model Agreement in Visual Saliency Modeling: A Comparative Study [J]. IEEE Transactions on Image Processing, 2013, 22 (1): 55-69.

[8] Marco Comuzzi, Jochem Vonk, Paul Grefen et al. Measures and mechanisms for process monitoring in evolving business networks [J]. Data \&amp; knowledge engineering, 2012, 71 (1): $1-28$.

[9] Meng, Shicong, Kashyap, Srinivas Raghav, Venkatramani, Chitra et al. Resource-Aware Application State Monitoring [J]. IEEE Transactions on Parallel and Distributed Systems: A Publication of the IEEE Computer Society, 2012, 23 (12): 2315-2329. 